\title{
One-Pot Four Component Reaction for the Synthesis of Formazans in an Environmentally Benign Procedure Mediated by $\mathrm{KHSO}_{4}$
}

\author{
Pranab Jyoti Das*, Jesmin Begum \\ Department of Chemistry, Gauhati University, Guwahati, India \\ Email: ${ }^{*}$ pjd23@rediffmail.com
}

Received 2 July 2015; accepted 15 August 2015; published 18 August 2015

Copyright (C) 2015 by authors and Scientific Research Publishing Inc.

This work is licensed under the Creative Commons Attribution International License (CC BY). http://creativecommons.org/licenses/by/4.0/

\section{(c) (i) Open Access}

\begin{abstract}
A microwave mediated solvent free one pot synthesis of formazans is developed using the solid acid, $\mathrm{KHSO}_{4}$. The products were obtained in a short reaction time in high yield. This study was undertaken to find an alternative and green method for the synthesis of formazans in the absence of corrosive mineral acids, buffered solutions and volatile organic compounds (VOCs).
\end{abstract}

\section{Keywords}

Formazan, One Pot Synthesis, Solvent Free, Four Component Reaction, Microwave, $\mathrm{KHSO}_{4}$

\section{Introduction}

Formazans were first reported by Von Pechmann [1] and by Bamberger [2]. They are intense coloured dyes characterized by their prominent $\pi-\pi^{*}$ transitions and these transitions are sensitive to the nature of the substituent present in the phenyl rings, nature of the organic solvents in solution and the acidity and basicity of the medium [3]-[5]. Extensive studies have been carried out relating to their structure evaluation, photochromatic transitions, tautomer formation, redox potential and organometallic chemistry [6]. Formazans also find extensive use in analytical chemistry [7] [8] and their photochemical and thermochemical properties have been investigated [9]. Formazans are reported to exhibit a wide spectrum of biological activities and notable among them are antiviral [10] [11], antimicrobial [12] [13], anti inflammatory, analgesic [14] antifungal [15] properties. Synthetic

${ }^{*}$ Corresponding author.

How to cite this paper: Das, P.J. and Begum, J. (2015) One-Pot Four Component Reaction for the Synthesis of Formazans in an Environmentally Benign Procedure Mediated by $\mathrm{KHSO}_{4}$. Green and Sustainable Chemistry, 5, 128-135.

http://dx.doi.org/10.4236/gsc.2015.53016 
methods for their preparation have been reviewed by Nineham earlier [16]. Their synthesis is based on two general procedures. The first is the reaction of aryldiazonium salts with phenylhydrazones of aldehydes in basic medium and the second type is based on coupling of aryldiazonium ions with active methylene groups followed by Japp Klingermann rearrangement [17]. However, these methods gave multiple products. Further to obtain the formazans in satisfactory yield strict control of $\mathrm{pH}$ of the medium is necessary. Newer methods of synthesis are reported and notable among them are the use of solid-liquid phase transfer catalysts [18]-[21], liquid-liquid systems and crown ethers [22], methods reported by Tezcan et al. [23]-[26] and a green method using solid Lewis acid namely nano $\mathrm{BF}_{3}-\mathrm{SiO}_{2}$ [27]. The commonly used method of synthesis involves three steps namely preparation of the aldehydehydrazones and the diazonium ions separately before reacting both in a suitable medium to give the formazan. This multiplicity of steps decreases the yield. Further diazotization is the key step and it requires the use of strong mineral acids and low temperature. The reaction conditions are stringent and require fine control of temperature as well as $\mathrm{pH}$. High cost of starting materials, use of corrosive mineral acids and volatile organic solvents makes the procedures unacceptable from the standpoint of green and sustainable chemistry. Further in some instances autocatalytic side reactions tend to lower the yield due to multiple products formation [27]. In some reported synthetic methods, alkali was used to accelerate the reaction which again makes the procedure unacceptable. While the only solvent free protocol reported by Bamoniri et al. [28] appears to be an improvement over other procedures; however, the use of $\mathrm{NaOH}$ and $\mathrm{BF}_{3}$-etherate in this method, prompts us to look for alternative green procedures which concomitantly result in high yields of the target product. It has already been reported that diazocoupling reactions could be conveniently carried in a one pot solvent free procedure using $\mathrm{KHSO}_{4}$ with microwave as the promoter in a green synthetic protocol [29]. The reported results encouraged us to extend this protocol and examined its applicability to the synthesis of formazans in a one pot solvent free four component reaction using this acidic salt.

\section{Results and Discussion}

We first examined the possibility by carrying out a one pot solvent free synthesis of formazans by mixing 4-nitroaniline, benzaldehyde and phenylhydrazine, $\mathrm{NaNO}_{2}, \mathrm{KHSO}_{4}$ and $2 \mathrm{~mL}$ of deionized water. The mixture was ground to a fine homogeneous product. Gradual development of an intense red colour during grinding lead us to conclude that the reaction could be performed by grinding only however on examining the product in prepared silicagel plates using petroleum ether (60 - 80): ethylacetate (9:1) as the eluent revealed only partial conversion. In order to drive the reaction to completion, the homogeneous mixture obtained after grinding, was subjected to microwave irradiation at 350 Watt power. Higher wattage of microwave was avoided as a precautionary measure against possible explosion because of the proven explosive proclivities of diazonium ions which are formed as the transient intermediate. Complete conversion to the formazan was observed after one minute of exposure. Encouraged by the results, we extended the reaction to the synthesis of a variety of formazans by using several combinations of aromatic aldehydes, aromatic amines and phenylhydrazone and in all cases yields were high and the products could be recovered in a simple work up procedure. The four component solvent free synthesis of formazans is shown in Scheme 1 and their physical characteristics summarized in Table 1.

A comparative study of the present methods to a few other procedures reported in literature is summarized in Table 2. Comparison reveals the superiority and better environmental acceptability of the one pot microwave mediated solvent free synthesis of formazans using cheap and easily available $\mathrm{KHSO}_{4}$.

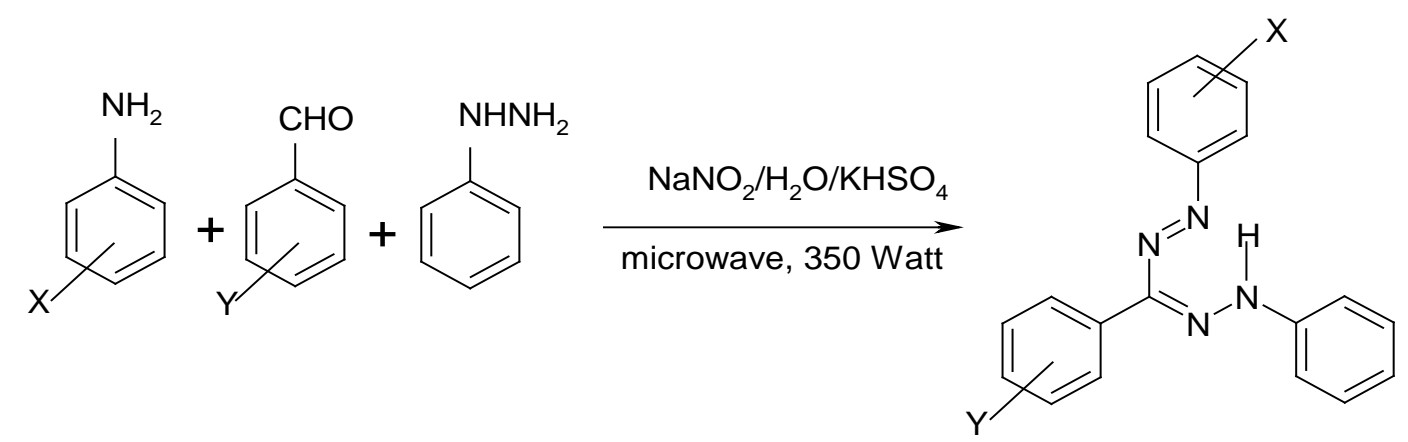

Scheme 1. Microwave mediated one pot solvent free synthesis of Formazans using $\mathrm{KHSO}_{4}$. 
Table 1. Physical characteristics of formazans obtained by a four component solid phase reaction using $\mathrm{KHSO}_{4}$.

\begin{tabular}{|c|c|c|c|c|c|c|}
\hline \multirow{2}{*}{ Entry } & \multirow{2}{*}{$\mathrm{X}$ (amine) R } & \multirow{2}{*}{$\mathrm{Y}$} & \multirow{2}{*}{ Yield (\%) } & \multirow{2}{*}{$\begin{array}{l}\text { Reaction } \\
\text { time (sec) }\end{array}$} & \multicolumn{2}{|c|}{ Melting points $\left({ }^{\circ} \mathrm{C}\right)$} \\
\hline & & & & & obs & lit \\
\hline 1 & $\mathrm{H}$ & $\mathrm{H}$ & 78 & 60 & $173-174$ & $172-174[21]$ \\
\hline 2 & $\mathrm{H}$ & $4-\mathrm{OCH}_{3}$ & 81 & 60 & $160-61$ & $157-59$ [21] \\
\hline 3 & $\mathrm{H}$ & $4-\mathrm{NO}_{2}$ & 70 & 60 & 199 & $196-98$ [27] \\
\hline 4 & $\mathrm{H}$ & $4-\mathrm{CH}_{3}$ & 72 & 60 & 157 & $153-155[27]$ \\
\hline 5 & 2-Cl & $\mathrm{H}$ & 68 & 60 & 146 & $142-143$ [23] \\
\hline 6 & $3-\mathrm{Cl}$ & $\mathrm{H}$ & 78 & 60 & $157-58$ & $158[23]$ \\
\hline 7 & $4-\mathrm{Br}$ & $\mathrm{H}$ & 67 & 60 & $190-91$ & $189-190[23]$ \\
\hline 8 & $4-\mathrm{Cl}$ & $\mathrm{H}$ & 65 & 60 & $120-121$ & 119 [23] \\
\hline 9 & $4-\mathrm{NO}_{2}$ & $\mathrm{H}$ & 81 & 60 & $232-234$ & $234-235[30]$ \\
\hline 10 & $3-\mathrm{NO}_{2}$ & $\mathrm{H}$ & 72 & 60 & 179 & $176[30]$ \\
\hline 11 & $2-\mathrm{NO}_{2}$ & $\mathrm{H}$ & 80 & 60 & 175 & $174-175[30]$ \\
\hline 12 & 2,4-diCl & $\mathrm{H}$ & 81 & 60 & 201 & 205 [30] \\
\hline 13 & $4-\mathrm{CH}_{3} \mathrm{CO}$ & $\mathrm{H}$ & 72 & 60 & $214-15$ & $214-216$ [30] \\
\hline 14 & $4-\mathrm{CH}_{3}$ & $\mathrm{H}$ & 65 & 60 & 156 & $153-154[30]$ \\
\hline 15 & 4-Cl & $4-\mathrm{CH}_{3}$ & 77 & 60 & $177-79$ & $179-183[30]$ \\
\hline 16 & $4-\mathrm{Cl}$ & $2-\mathrm{CH}_{3} \mathrm{O}$ & 82 & 60 & $180-182$ & 180 - 181 [29] \\
\hline 17 & $4-\mathrm{CH}_{3} \mathrm{O}$ & $2-\mathrm{Cl}$ & 65 & 60 & $203-205$ & $203-206$ [30] \\
\hline 18 & $4-\mathrm{CH}_{3}$ & $4-\mathrm{NO}_{2}$ & 71 & 60 & $188-190$ & $188-190$ [30] \\
\hline 19 & $4-\mathrm{NO}_{2}$ & $4-\mathrm{NO}_{2}$ & 78 & 60 & $208-211$ & 209 [30] \\
\hline
\end{tabular}

Table 2. Comparison of different methods of the synthesis of formazans.

\begin{tabular}{ccccccc}
\hline Entry & Reagents used & Yield & Reaction time & Reaction steps & Remarks & Ref. \\
\hline 1 & $\mathrm{PTC} / \mathrm{M}_{2} \mathrm{CO}_{3} / \mathrm{CH}_{2} \mathrm{Cl}_{2}, \mathrm{M}=\mathrm{K}, \mathrm{Na}$ & $45 \%-70 \%$ & $1-4 \mathrm{hrs}$ & 3 & VOC used & {$[22]$} \\
2 & $\mathrm{BF}_{3}-\mathrm{SiO}_{2}$ & $78 \%-88 \%$ & $1-2 \mathrm{mins}$ & 2 & Solvent free & {$[28]$} \\
3 & Mineral acid, $\mathrm{NaOH}, \mathrm{CH}_{3} \mathrm{COONa}$ & $80 \%$ & $3.5 \mathrm{hrs}$ & 3 & VOC used & {$[31]$} \\
4 & alkali & $54 \%-75 \%$ & - & 3 & Temp and pH control & {$[6]$} \\
5 & Amberlite IR120 $\left(\mathrm{Na}^{+}\right)$, acid, cyclohexane & $65 \%-74 \%$ & $1-2 \mathrm{hrs}$ & 3 & VOC used & {$[30]$} \\
\hline
\end{tabular}

\section{Conclusion}

In all the previously reported synthesis of formazan, either the aldehyde phenylhydrazones or the diazonium ions or both were prepared separately and brought into reaction in a suitable medium. In this work, the possibility of a one pot procedure in the absence of any organic solvent or added coupling agents was explored. The use of moist $\mathrm{KHSO}_{4}$ resulted in a one pot environmentally benign synthesis of formazans in high yield and in a very short reaction time. Further, delicate control of temperature as well as $\mathrm{pH}$ was not necessary making the procedure suitable for industrial application for large scale environmentally benign production of formazans dyes.

\section{Experimental Section}

\subsection{General}

Chemicals were purchased from Loba chieme (India) and purified by procedures reported in literature [32]. 
Formazans obtained were confirmed by comparing their melting points with those reported in literature. Melting points were recorded in open capillaries and are uncorrected. Products were purified by repeated column chromatography. UV-vis spectra were recorded in UV-1800 Shimadzu UV spectrophotometer, IR spectra were recorded in $\mathrm{KBr}$ pellets in a Perkin Elmer FT-IR 1600 spectrophotometer and ${ }^{1} \mathrm{H}$ and ${ }^{13} \mathrm{C}$ NMR were recorded in Bruker Bio Spin $300 \mathrm{MHz}$ spectrometer using $\mathrm{CDCl}_{3}$ as solvent and TMS as internal standard. Mass spectra of new compounds were recorded in Micromass QTOF ESI-MS instrument (model HAB273) and Microwave irradiation of reaction mixture was performed in reactor procured from Catalyst ${ }^{\mathrm{TM}}$ (India).

\subsection{General Procedure for Synthesis of Formazan in the Solid Phase}

A mixture of aromatic aldehyde $(1 \mathrm{mmol})$, phenylhydrazine $(1 \mathrm{mmol})$, aromatic amine $(1 \mathrm{mmol})$ and $\mathrm{NaNO}_{2}$ (1.2 mmol), $10 \mathrm{~mol} \%$ of $\mathrm{KHSO}_{4}$ and $2 \mathrm{~mL}$ of deionized water was ground to a homogeneous mixture. The bright coloured mixture was exposed to microwave irradiation (350 Watt) for $60 \mathrm{sec}$. After completion of the reaction, the solid mass obtained was washed with water till free of $\mathrm{KHSO}_{4}$. The remaining solid was dissolved in ethylacetate, dried using anhydrous $\mathrm{Na}_{2} \mathrm{SO}_{4}$ and product obtained by reduced pressure evaporation of the solvent. Finally the products were recrystallized from 95\% (v/v) EtOH. All formazans synthesized gave characteristics $\pi-\pi^{*}$ absorption in the visible region at 350 to $450 \mathrm{~nm}$ which on oxidation with dil $\mathrm{HNO}_{3}$ at room temperature or with $5 \% \mathrm{KMnO}_{4}$ solution, shifted the absorption maxima to around $300 \mathrm{~nm}$ or below indicating their conversion to tetrazolium salts [5]. This was a confirmative test for the formation of formazans as the product. Table 3 summarizes the shift in the UV absorption maxima of selected formazans after oxidation leading to the formation of tetrazolium salts. The UV spectra of selected formazans before and after oxidation shown in Figure 1 and Figure 2, a and b respectively.

IR spectra of formazans gave the characteristic absorption at $1410-1420 \mathrm{~cm}^{-1}$ which is characteristic of the azo group. Several aromatic aldehydes with both electron withdrawing as well as electron releasing groups were examined and the reaction time as well as the yields in all cases were observed to be independent of the electronic effects of the groups present in the benzene rings of both the aromatic amine as well as the aldehyde. We have thus demonstrated that formazans could be successfully synthesized in a one pot procedure starting from arylaldehydes, arylamines, phenylhydrazine and sodium nitrite promoted by $\mathrm{KHSO}_{4}$ without having to synthesize the hydrazones or the diazonium ions separately. The reaction could be carried out without acids or bases consequently control of $\mathrm{pH}$ and reaction temperature was not necessary. The yields were high vis-a-vis the wet reaction where several by products are usually obtained resulting in low yield. Further, the present synthetic protocol appears to be more economical and less harmful from environmental point of view. It is expected that this solvent free protocol will be ideally suited for large scale industrial production of a variety of formazan dyes at reduced cost. The physical and spectral data of some selected formazans are listed as the following.

\begin{tabular}{|c|c|c|}
\hline Compound & $\mathrm{UV} \lambda_{\max }$ in $95 \%(\mathrm{v} / \mathrm{v}) \mathrm{EtOH}$ & UV $\lambda_{\max }$ after Oxid ${ }^{\mathrm{n}}$ \\
\hline Entry 9 & $413 \mathrm{~nm}$ & $307 \mathrm{~nm}$ \\
\hline Entry 10 & $341 \mathrm{~nm}$ & $308 \mathrm{~nm}$ \\
\hline Entry 11 & $331.91 \mathrm{~nm}$ & $310 \mathrm{~nm}$ \\
\hline Entry 12 & $372 \mathrm{~nm}$ & $305 \mathrm{~nm}$ \\
\hline Entry 13 & $371 \mathrm{~nm}$ & $310 \mathrm{~nm}$ \\
\hline Entry 14 & $372 \mathrm{~nm}$ & $315 \mathrm{~nm}$ \\
\hline Entry 15 & $348 \mathrm{~nm}$ & $300 \mathrm{~nm}$ \\
\hline Entry 16 & $355 \mathrm{~nm}$ & $312 \mathrm{~nm}$ \\
\hline Entry 17 & $353 \mathrm{~nm}$ & $310 \mathrm{~nm}$ \\
\hline Entry 18 & $426 \mathrm{~nm}$ & $404 \mathrm{~nm}$ \\
\hline Entry 19 & $402 \mathrm{~nm}$ & $354 \mathrm{~nm}$ \\
\hline
\end{tabular}




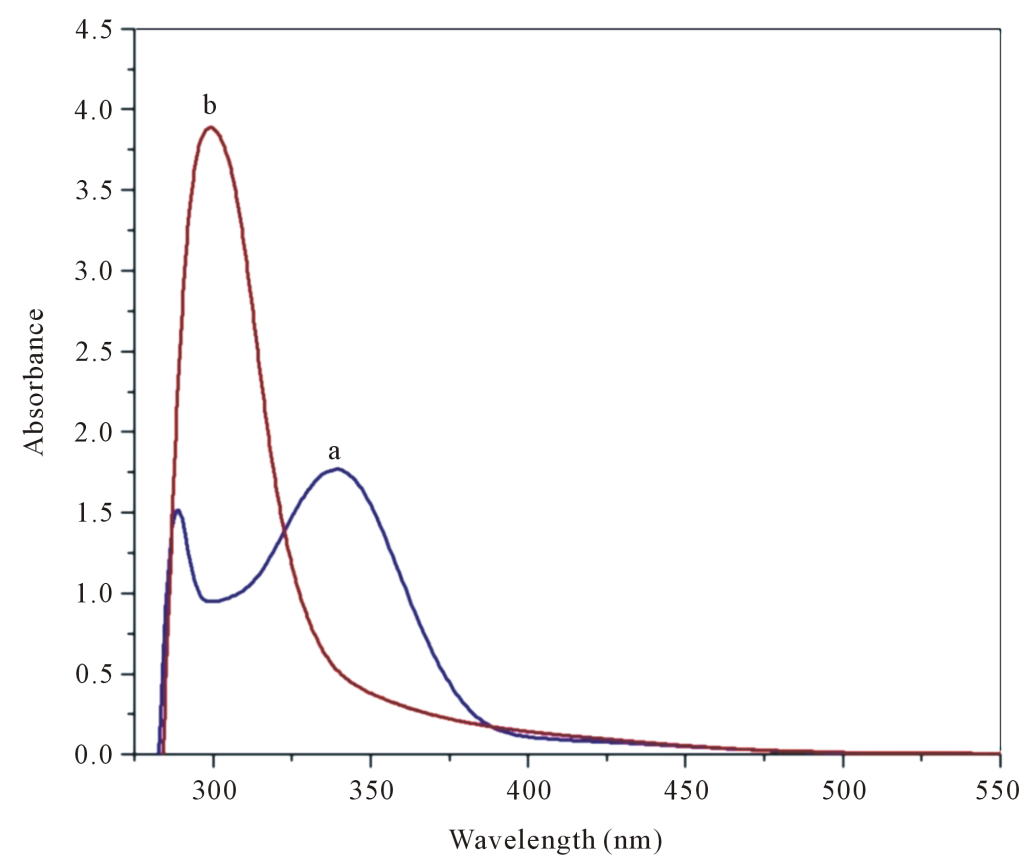

$\mathrm{a} \longrightarrow$ 1-(3-nitrophenyl)-3,5-diphenyl formazan $(341.02 \mathrm{~nm})$
$\mathrm{b}-($ - 3 -nitrophenyl)-3,5-diphenyl formazan $(308.19 \mathrm{~nm})$
(after oxidation)

Figure 1. UV max of formazan (entry 10) before and after oxidation in ethanol solution.

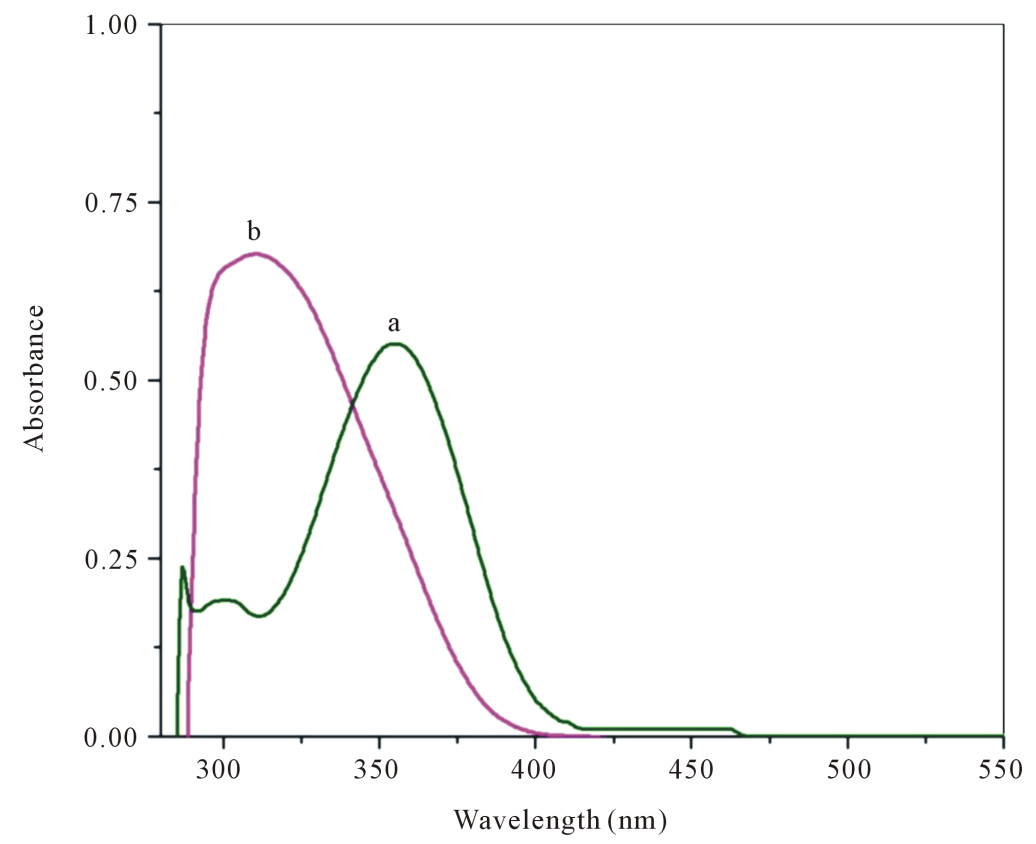

a - 1-(4-methoxyphenyl)-3-(2-chlorophenyl)-5-phenyl formazan (353.18 nm)

b-1-(4-methoxyphenyl)-3-(2-chlorophenyl)-5-phenyl formazan $(310.59 \mathrm{~nm})$ (after oxidation)

Figure 2. UV max of formazan (entry 17) before and after oxidation in ethanol solution. 
1-(4-nitrophenyl)-3,5-diphenylformazan (Entry 9): dark red, UV(EtOH): $\lambda_{\max } 413$ (K-band), IR (KBr): $v \mathrm{~cm}^{-1}$ 3094 (Ar-C-H), $2970(\mathrm{~N}-\mathrm{H}), 1651$ (-C=C-), $1556\left(-\mathrm{NO}_{2}\right), 1415(-\mathrm{N}=\mathrm{N}-), 1303\left(-\mathrm{NO}_{2}\right){ }^{1} \mathrm{H}-\mathrm{NMR}(300 \mathrm{MHz}$ $\left.\left.\mathrm{CDCl}_{3}\right): \delta_{\mathrm{H}} \operatorname{ppm} 6.979(1 \mathrm{H}, \mathrm{d} \mathrm{J}=7.6 \mathrm{~Hz}, \mathrm{NH}), 7.140-8.496(\mathrm{~m}, 14 \mathrm{H}, \mathrm{Ar}-\mathrm{H})\right),{ }^{13} \mathrm{C}$ NMR $\left(75 \mathrm{MHz}, \mathrm{CDCl}_{3}\right): \delta$ ppm. 114.72, 118.28, 120.43, 121.9, 123.48, 125.18, 127.99, 128.57, 132.37, 133.58, 134.31, 145.19, 150.01 HRMS (ESI): 344.612 calc. 243. HRMS (ESI): 346.128 (M+) calc. 345.

1-(3-nitrophenyl)-3,5-diphenylformazan (Entry 10): dark red, UV(EtOH): $\lambda_{\max } 341 \mathrm{~nm}$ (K-band), IR (KBr): $v$ $\mathrm{cm}^{-1} 3000$ (N-H str), 2369 (C-H str), 1653 (-C=C-), $1548\left(-\mathrm{NO}_{2}\right), 1417(-\mathrm{N}=\mathrm{N}-), 1302\left(-\mathrm{NO}_{2}\right),{ }^{1} \mathrm{H}-\mathrm{NMR}$ (300 $\left.\mathrm{MHz}, \mathrm{CDCl}_{3}\right): \delta_{\mathrm{H}} \mathrm{ppm} 7.227(1 \mathrm{H}, \mathrm{s}, \mathrm{N}-\mathrm{H}), 7.253-8.025(14 \mathrm{H}, \mathrm{m}, \mathrm{Ar}-\mathrm{H}),{ }^{13} \mathrm{CNMR}\left(75 \mathrm{MHz} \mathrm{CDCl}_{3}\right): \delta \mathrm{ppm}$ 112.30, 112.30, 113.24, 114.49, 116.98, 119.00, 126.52, 128.73, 129.19, 129.93, 138.44, 139.52, 143.58, 145.19, HRMS (ESI): $346.128\left(\mathrm{M}^{+}\right)$calc. 345.35.

1-(2-nitrophenyl)-3,5-diphenylformazan (Entry 11): red, UV(EtOH): $\lambda_{\max } 331.91 \mathrm{~nm}$ (K-band), R (KBr): $v$ $\mathrm{cm}^{-1} 3346$ (Ar-C-H), $3086(\mathrm{~N}-\mathrm{H}), 1648(-\mathrm{C}=\mathrm{C}-), 1555\left(-\mathrm{NO}_{2}\right), 1419(-\mathrm{N}=\mathrm{N}-), 1302\left(-\mathrm{NO}_{2}\right),{ }^{1} \mathrm{H}-\mathrm{NMR}(300 \mathrm{MHz}$, $\left.\mathrm{CDCl}_{3}\right): \delta_{\mathrm{H}}$ ppm $6.883(1 \mathrm{H}, \mathrm{t}, \mathrm{J}=7.8, \mathrm{~J}=7.5, \mathrm{NH}), 7.759-8.203(14 \mathrm{H}, \mathrm{m}, \mathrm{Ar}-\mathrm{H}),{ }^{13} \mathrm{C}$ NMR $\left(75 \mathrm{MHz}, \mathrm{CDCl}_{3}\right)$ : $\delta$ ppm. 116.47, 118.49, 121.01, 126.19, 127.25, 129.02, 130.04, 134.49, 136.34, 137.02, 142.16, 143.9, 147.84, 149.34, HRMS (ESI): 344.612 obs, 345 calc.

1-(2,4-dichlorophenyl)-3,5-diphenylformazan (Entry 12): dark red, UV (EtOH): $\lambda_{\max } 374.96 \mathrm{~nm}$ (K band), IR (KBr): $v \mathrm{~cm}^{-1} 3276$ (C-H str), 3000 (N-H str), 1642 (-C=C-), $1556(\mathrm{C}-\mathrm{Cl}), 1414(-\mathrm{N}=\mathrm{N}-),{ }^{1} \mathrm{H}-\mathrm{NMR}(300 \mathrm{MHz}$, $\left.\mathrm{CDCl}_{3}\right): \delta_{\mathrm{H}}$ ppm $7.193(1 \mathrm{H}, \mathrm{s}, \mathrm{NH}) 7.612-7.639(13 \mathrm{H}, \mathrm{m}, \mathrm{Ar}-\mathrm{H}),{ }^{13} \mathrm{C} \mathrm{NMR}\left(\mathrm{CDCl}_{3}, 75 \mathrm{MHz}\right): 77.41115 .43$, 118.95, 123.20, 123.62, 128.41, 128.98, 129.47, 129.70, 130.33, 130.56, 130.78, 131.07, 133.49, 145.12, HRMS (ESI): 369.0617 (M+ Obs) 369.25 (Calc.).

1-(4-acetoxyphenyl)-3,5-diphenylformazan (Entry 13): dark red, UV (EtOH): $\lambda_{\max } 371$ (K-band), IR (KBr): $v$ $\mathrm{cm}^{-1} 2987$ (N-H str), 2351 (aro C-H), 1658 (-CO-), 1417 (-N=N-), ${ }^{1} \mathrm{H}-\mathrm{NMR}$ (300 MHz, $\mathrm{CDCl}_{3}$ ): $\delta_{\mathrm{H}} \mathrm{ppm} 6.619$ $(1 \mathrm{H}, \mathrm{s}, \mathrm{N}-\mathrm{H}), 7.648$ - $7.681(14 \mathrm{H}, \mathrm{m}, \mathrm{Ar}-\mathrm{H}), 2.524\left(3 \mathrm{H},-\mathrm{COCH}_{3}\right){ }^{13} \mathrm{C} \mathrm{NMR}\left(\mathrm{CDCl}_{3}, 75 \mathrm{MHz}\right): 26.03,112.59$, 113.60, 119.87, 126.05, 127.50, 128.27, 128.50, 129.20, 130.75, 135.25, 137.18, 144.60, 151.19, 196.73 HRMS (ESI): $342.32\left(\mathrm{M}^{+}\right.$, Obs) 342.39 (Calc).

1-(4-methylphenyl)-3,5-diphenylformazan (Entry 14): dark red, UV(EtOH): $\lambda_{\max } 346.23$ (K-band), IR (KBr): $v$ $\mathrm{cm}^{-1} 2994(\mathrm{~N}-\mathrm{H}), 1644(-\mathrm{C}=\mathrm{C}-), 1556(-\mathrm{C}=\mathrm{N}-), 1412(-\mathrm{N}=\mathrm{N}-),{ }^{1} \mathrm{H}-\mathrm{NMR}\left(300 \mathrm{MHz}, \mathrm{CDCl}_{3}\right) \delta_{\mathrm{H}} \mathrm{ppm} 2.258(3 \mathrm{H}$, s), $6.625(1 \mathrm{H}, \mathrm{d}, \mathrm{J}=8.4, \mathrm{NH}), 7.004-8.24(14 \mathrm{H}, \mathrm{m}, \mathrm{Ar}-\mathrm{H}),{ }^{13} \mathrm{C}$ NMR $\left(75 \mathrm{MHz}, \mathrm{CDCl}_{3}\right) \delta \mathrm{ppm} 146.81,143.55$, 141.76, 133.73, 129.92, 129.72, 129.38, 129.22, 127.88, 126.12, 124.03, 123.94, 123.56, 121.1, 115.31, 113, 20.42. HRMS (ESI): 344.612 (Obs) 243 (Calc.).

1-(4-chlorophenyl)-3-(4-methylphenyl)-5-phenyl formazan (Entry 15): orange red, UV(EtOH): $\lambda_{\max } 355.12$ nm (K-band), IR (KBr): $v \mathrm{~cm}^{-1}$ 3001(N-H str), 2364 (C-H str), 1650 (-C=C-), 1414 (-N=N-), ${ }^{1} \mathrm{H}-\mathrm{NMR}$ (300 $\left.\mathrm{MHz}, \mathrm{CDCl}_{3}\right): \delta_{\mathrm{H}} \mathrm{Ppm} 1.798\left(3 \mathrm{H}, \mathrm{s}, \mathrm{CH}_{3}\right), 6.622(1 \mathrm{H}, \mathrm{s}, \mathrm{N}-\mathrm{H}) 6.650-7.627(13 \mathrm{H}, \mathrm{m}, \mathrm{Ar}-\mathrm{H}),{ }^{13} \mathrm{CNMR}(75 \mathrm{MHz}$, $\left.\mathrm{CDCl}_{3}\right): \delta \mathrm{ppm} 20.13,117.29,118.59,122.27,124.65,126.80,127.60,28.81,130.05,132.11,137.25,138.97$, 144.62, 151.12, HRMS (ESI): 349.872 (Obs) 348.5 (Calc).

1-(4-chlorophenyl)-3-(2-methoxyphenyl)-5-phenylformazanc (Entry 16): dark red, UV (EtOH): $\lambda_{\max } 353.18$ nm (K-band), IR (KBr): $v \mathrm{~cm}^{-1} 2923(\mathrm{~N}-\mathrm{H}), 1651$ (-C-O-), $1556(-\mathrm{C}=\mathrm{N}), 1419(-\mathrm{N}=\mathrm{N}-),{ }^{1} \mathrm{H}-\mathrm{NMR}(300 \mathrm{MHz}$, $\left.\mathrm{CDCl}_{3}\right): \delta_{\mathrm{H}} \mathrm{ppm} 3.933(3 \mathrm{H}, \mathrm{d}, \mathrm{J}=13.4), 6.559(1 \mathrm{H}, \mathrm{s}, \mathrm{N}-\mathrm{H}), 6.587-8.125(13 \mathrm{H}, \mathrm{m}, \mathrm{Ar}-\mathrm{H}){ }^{13} \mathrm{CNMR}(75 \mathrm{MHz}$, $\left.\mathrm{CDCl}_{3}\right): \delta \mathrm{ppm} 50.02,112.72,20.28,126.43,126.80,128.28,128.99,129.25,129.57,132.37,132.58,133.31$, 144.19, 147.58, 148.79, HRMS (ESI): $349.321\left(\mathrm{M}^{+}\right)$obs, 348.5 calc.

1-(4-methoxyphenyl)-3-(2-chlorophenyl-5-phenylformazan (Entry 17): dark red, UV (EtOH): $\lambda_{\max } 353.18$ (K-band), IR (KBr): $v \mathrm{~cm}^{-1} 2985$ (N-H), 2363 (-C-H), 1651 (C-O) 1599 (C=N str), 1415 (-N=N-) ${ }^{1} \mathrm{H}-\mathrm{NMR}(300$ $\left.\mathrm{MHz}, \mathrm{CDCl}_{3}\right): \delta_{\mathrm{H}} \mathrm{ppm} 2.956\left(3 \mathrm{H}, \mathrm{s}, \mathrm{OCH}_{3}\right), 6.925(\mathrm{~s}, \mathrm{~N}-\mathrm{H}), 6.950-7.489(13 \mathrm{H}, \mathrm{m}, \mathrm{Ar}-\mathrm{H}),{ }^{13} \mathrm{CNMR}(75 \mathrm{MHz}$, $\left.\mathrm{CDCl}_{3}\right) \delta$ ppm 50.02, 112.72, 120.28, 126.43, 126.80, 128.28, 128.99, 129.57, 129.25, 132.37, 132.58, 133.31, 144.19, 147.58, 148.79. HRMS (ESI): 376.1 calc. 376.84.

1-(4-methylphenyl)-3-(4-nitrophenyl)-5-phenyl formazan (Entry 18): UV (EtOH): $\lambda_{\max } 404.21 \mathrm{~nm}$ (K-band), IR (KBr): $v \mathrm{~cm}^{-1} 2968$ (N-H str), 1648 (-C=C-), $1540\left(-\mathrm{NO}_{2}\right), 410(-\mathrm{N}=\mathrm{N}-), 1301\left(-\mathrm{NO}_{2}\right),{ }^{1} \mathrm{H}-\mathrm{NMR}(300 \mathrm{MHz}$, $\left.\mathrm{CDCl}_{3}\right): \delta_{\mathrm{H}}$ ppm $2.568\left(3 \mathrm{H}, \mathrm{s},-\mathrm{CH}_{3}\right), 6.625(1 \mathrm{H}, \mathrm{s}, \mathrm{N}-\mathrm{H}), 6.653-8.240(13 \mathrm{H}, \mathrm{m}, \mathrm{Ar}-\mathrm{H}),{ }^{13} \mathrm{CNMR}(75 \mathrm{MHz}$, $\left.\mathrm{CDCl}_{3}\right): \delta \mathrm{ppm} 20.42,77.43,113.00,115.31,121.10,123.56,123.94,124.03,126.12,127.88,129.22,129.92$, 133.73, 141.76, 143.55, 146.81. HRMS (ESI): 360.12 ( $\mathrm{M}^{+}$) obs, 359 (calc).

1-(4-nitrophenyl)-3-(4-nitrophenyl)-5-phenyl formazan (Entry 19): red, UV (EtOH): $\lambda_{\max } 354.77$ (K-band), IR (KBr): $v \mathrm{~cm}^{-1} 2988$ (N-H str), $1653(-\mathrm{C}=\mathrm{C}-), 1548\left(-\mathrm{NO}_{2}\right), 1418(-\mathrm{N}=\mathrm{N}-), 1299\left(-\mathrm{NO}_{2}\right),{ }^{1} \mathrm{H}-\mathrm{NMR}(300 \mathrm{MHz}$, $\left.\mathrm{CDCl}_{3}\right): \delta_{\mathrm{H}} \mathrm{ppm} 6.612(1 \mathrm{H}, \mathrm{s}, \mathrm{N}-\mathrm{H}), 6.64-8.174(13 \mathrm{H}, \mathrm{m}, \mathrm{Ar}-\mathrm{H}),{ }^{13} \mathrm{C}$ NMR $\left(75 \mathrm{MHz}, \mathrm{CDCl}_{3}\right): \delta \mathrm{ppm} 77.91$, 
113.66, 117.86, 121.82, 127.76, 128.43, 129.42, 130.00, 130.78, 136.08, 138.21, 147.85, 151.05, 152.08. HRMS ESI): 391.21 (M obs) 390 (calc.)

\section{Acknowledgements}

The authors are thankful to the HOD, Department of Chemistry, Gauhati University, for providing laboratory facilities.

\section{References}

[1] Pechmann, H. (1892) Ueber die Einwirkung von Diazobenzol auf Malonsäure. Berichte, 25, 3175.

[2] Bamberger, E. and Wheelwright, E.W. (1892) Ueber die Einwirkung von Diazobenzol auf Acetessigäther. Berichte, 25, 3201.

[3] Sanli, S., Sanli, N. and Alsancak, G. (2009) Determination of Prootonation Constants of Some Tetracycline Antibiotics by Potentiommetry and LC Methods in Water and Acetinitrile-Water Binary Mixtures. Journal of the Brazilian Chemical Society, 20, 939-946.

[4] Beltran, J.L., Sanli, N., Fonrodona, G., Barren, D., Özkan, G. and Barbosa, J. (2003) Spectrophotometric, Potentiometric and Chromatographic $\mathrm{pK}_{\mathrm{a}}$ Values of Polyphenolic Acids in Water and Acetonitrile-Water Media. Analytica Chimica Acta, 484, 253-264. http://dx.doi.org/10.1016/S0003-2670(03)00334-9

[5] Senoz, H. (2012) The Chemistry of Formazans and Tetrazolium Salts. Hacettepe Journal of Biology and Chemistry, 40, 293-301.

[6] Tezcan, H. and Ozbek, N. (2005) The Synthesis of Some Bis-Substituted Formazans and the Investigation of the Effect of Substituent upon Their UV-Vis Absorption Values. Communications Faculty of Sciences University of Ankara Series $B, \mathbf{5 1}, 13-28$.

[7] Debnam, P.M. and Shearer, G. (1997) Colorimetric Assay for Substrates of NADP ${ }^{+}$-Dependent Dehydrogenase Based on Reduction of Tetrazolium Dyes to Its Soluble Formazans. Analytical Biochemistry, 250, 253-255. http://dx.doi.org/10.1006/abio.1997.2245

[8] Barsoum, B.N., Khella, S.K., Elwaby, A.H.M., Abbas, A.A. and Ibrahim, Y.A. (1998) In Evaluation of Some New 14and 15-Crown Formazans as Carriers in Cesium Ion Selective Electrodes. Talanta, 47, 1215. http://dx.doi.org/10.1016/S0039-9140(98)00204-5

[9] Van Aardt, J.H.P., Tadros, W., Cottrell, H.J., Pain, D.L., Slack, R., Brand, J.C.D., Minkoff, G.J., Mulcahy, M.F.R., Watt, I.C., Ward, E.R., Coulson, T.M., Hawkins, J.W., Balenovic, K., Dvornik, D., Blanchard, J.P., Goering, H.L., Lane, E.S. and Williams, C. (1954) Some Heptatriene Derivatives. Journal of the Chemical Society, 2967.

[10] Misra, V.S., Dhar, S. and Chowdhary, B.L. (1978) Synthesis of Some Newer Formazans and Tetrazolium Salts as Antiviral Agents. Pharmazie, 33, 790-792.

[11] Mukherjee, D.D., Shukla, S.K. and Chowdhary, B.L. (1981) Synthesis of Some New Formazans as Potential Anti Viral Agents. Archiv der Pharmazie, 314, 991-994. http://dx.doi.org/10.1002/ardp.19813141204

[12] Desai, R.M. and Desai, J.M. (1999) Synthesis and Antimicrobial Activity of Some New Formazan Derivatives. Indian Journal of Heterocyclic Chemistry, 8, 329-331.

[13] Samel, A.B. and Pai, N.R. (2010) Synthesis and Antimicrobial Activity of Some Novel Formazan Derivatives. Journal of Chemical and Pharmaceutical Research, 2, 60-67.

[14] Kalsi, R., Pande, K., Bhalla, T.N., Parmar, S.S. and Brathwal, J.P. (1988) Novel Formazans as Potent Anti-Inflammatory and Analgesic Agents. Pharmacology, 37, 218-224. http://dx.doi.org/10.1159/000138469

[15] Desai, K.G. and Desai, K.R. (2006) Microbial Screening of Novel Synthesized Formazans Having Amide Linkages $\varphi$. Journal of Heterocyclic Chemistry, 43, 1083-1089. http://dx.doi.org/10.1002/jhet.5570430440

[16] Nineham, A.W. (1955) The Chemistry of Formazans and Tetrazolium Salts. Chemical Reviews, 5, 355-483. http://dx.doi.org/10.1021/cr50002a004

[17] Ashley, J.N., Davis, B.M., Nineham, A.W. and Slack, R. (1953) Tetrazolium Compounds. Part I. Tetrazolium Compounds Containing Substituted Phenyl and Heterocyclic Rings. Journal of the Chemical Society, 1953, 3881-3888. http://dx.doi.org/10.1039/jr9530003881

[18] Korzeniowski, S.H. and Gokel, G.W. (1977) Crown Cation Complex Effect. V. Reaction of Aryldiazonium Ions in Non Polar Media. Tetrahedron Letters, 18, 1637-1640. http://dx.doi.org/10.1016/S0040-4039(01)93236-8

[19] Tezcan, H. (2008) Synthesis and Spectral Properties of Some Bis-Substituted Formazans. Spectrochimica Acta Part A, 69, 971-979. http://dx.doi.org/10.1016/j.saa.2007.05.061 
[20] Iwamoto, H., Yoshimura, M., Sonoda, T. and Kobayashi, H. (1983) Anion-Catalyzed Phase-Transfer Catalysis. I. Application to Diazo-Coupling Reactions. Bulletin of the Chemical Society of Japan, 56, 796-801. http://dx.doi.org/10.1246/bcsj.56.796

[21] Hashida, Y., Kubota, K. and Sekiguchi, S. (1988) Phase-Transfer-Catalyzed Azo Coupling Reactions in Two-Phase Systems. Bulletin of the Chemical Society of Japan, 61, 905-909. http://dx.doi.org/10.1246/bcsj.61.905

[22] Katritzky, A.R., Belyakov, S.A., Cheng, D. and Durst, H.D. (1995) Syntheses of Formazans under Phase-Transfer Conditions. Synthesis, 1995, 577-581. http://dx.doi.org/10.1055/s-1995-3958

[23] Tezcan, H. and Uzluk, E. (2007) The Synthesis and Spectral Properties Determination of 1,3-Substituted Phenyl-5phenylformazans. Dyes and Pigments, 75, 633-640. http://dx.doi.org/10.1016/j.dyepig.2006.07.011

[24] Tezcan, H., Uzluk, E. and Aksu, M.L. (2008) Electrochemical and Structural Properties of 1,3-Substituted (-Cl, -Br) Phenyl-5-Phenylformazans. Journal of Electroanalytical Chemistry, 619-620, 105-116. http://dx.doi.org/10.1016/j.jelechem.2008.03.013

[25] Tezcan, H., Can, S. and Tezcan, R. (2002) The Synthesis and Spectral Properties Determination of 3-Substituted-1,5diphenylformazans. Dyes and Pigments, 52, 121-127. http://dx.doi.org/10.1016/S0143-7208(01)00074-2

[26] Tezcan, H. and Ozkan, N. (2003) Substituent Effect on the Spectral Properties of Some 3-Substituted Formazans. Dyes and Pigments, 56, 159-166. http://dx.doi.org/10.1016/S0143-7208(02)00131-6

[27] Spande, T.F. and Glenner, G. (1973) Reactions of Indole with Diazonium Salts (Fast Red B). Journal of the American Chemical Society, 95, 3400-3402. http://dx.doi.org/10.1021/ja00791a063

[28] Bamoniri, A., Mirjalili, B.B.F. and Arani, N.M. (2014) Nano $\mathrm{BF}_{3} \cdot \mathrm{SiO}_{2}$ : A Green Heterogeneous Solid Acid for Synthesis of Formazan Dyes under Solvent Free Condition. Journal of Molecular Catalysis A: Chemical, 393, 272-278.

[29] Jin, J., Wen, Z., Long, J., Wang, Y., Matsuura, T. and Meng, J. (2000) One-Pot Diazo Coupling Reaction under Microwave Irradiation in the Absence of Solvents. Synthetic Communications, 30, 829-834. http://dx.doi.org/10.1080/00397910008087094

[30] Das, P.J. and Begum, J. (2015) Microwave Mediated Solvent Free Synthesis of Formazans Catalyzed by Simple Ionic Liquids Derived from Dialkylammonium Salts. RSC Advances, 5, 44604-44609. http://dx.doi.org/10.1039/C5RA06363A

[31] Tezcan, H. and Ekmekci, G. (2010) Electrochemical and Spectroscopic Behaviours of 1-(o-, m-, $p$-Cl or -Br) Substituted Phenyl-3,5-Diphenylformazans in Dimethylsulphoxide. Acta Chimica Slovenica, 57, 189-197.

[32] Furniss, B.S., Hannaford, A.J., Smith, P.W.G. and Tatchell, A.R., Eds. (2007) Vogel’s Textbook of Practical Organic Chemistry. 5th Edition, Pearson Education, London. 